\title{
Communities of practice environment
}

\author{
J. Feldman, D. Lee \& D. Thaw \\ International Computer Science Institute, Berkeley, CA, USA
}

\begin{abstract}
The Communities of Practice Environment (CoPE) project is an exploration of the potential for extending the social processes of the open source software movement to a wide range of other cooperative activities. The general ideas are best expressed in Steven Weber's book: The Success Of Open Source, Harvard U. Press, 2004. Obviously enough, collaboration over the Internet is a core factor in the development of open source software by diverse and distributed individuals and groups. But these developers are all IT sophisticates - could people without such expertise also form effective distributed communities of practice? We have built and deployed a platform that explores this possibility. The CoPE project provides web-based support for formal and informal groups to democratically work together and decide upon actions of common interest. The workflow in a CoPE is organized around documents with group discussion and decision making involving these documents. A CoPE can be set up and operated without requiring any IT expertise. CoPE sites have been used by groups ranging from scientific conferences and university departments to multilingual community organizations. This paper describes the CoPE system http://cope.icsi.berkeley.edu and our experience with its use.

Keywords: community of practice, collaboration, open source, content management, workflow, discussion, information technology, computer supported collaborative work, CoPE.
\end{abstract}

\section{Introduction}

The use of web-based collaboration systems can greatly enhance the productivity of groups or organizations, especially if the group is distributed and it is difficult for group members to meet physically. CoPE is a system that facilitates cooperative work. It provides information management, facilitates communication within the group, and can help instil a sense of community. 
Many groups cannot take advantage of web-based collaboration systems because of the high cost and/or the lack of technical expertise. Setting up and maintaining one of these systems is not a trivial task.

Most groups have four options when it comes to web-based collaboration systems: 1. Purchase a commercial product such as Microsoft Sharepoint, which provides advanced capabilities but is very costly. 2. Use free web-based groupware such as Yahoo! Groups or MSN Communities, which are easy to use but lack functionality and customizability. 3. Download and install an open source product, such as Plone, which is free of cost and customizable but requires advanced technical knowledge. 4. Employ a variety of Wiki, which is fairly easy to use, but provides no structure. All of the four options above have tradeoffs that most groups are not willing or able to make. The Communities of Practice Environment (CoPE) project aims to provide a web-based collaboration environment that doesn't involve these negative tradeoffs. The goal is for it to be highly customizable and functional, free of cost, and easy to setup and use without requiring IT expertise.

\section{Description of the CoPE system}

CoPE is a web-based collaboration system that is centred on documents. Information is represented in the form of a document such as a Microsoft Word document, PDF Document, or text. The CoPE system is designed to support the creation of documents, sharing them with the group, discussing a document, and ultimately making decisions on it as a group. This process is captured through a workflow where the document goes through different states as it progresses. Another feature of CoPE is that it can be customized to the group's needs through selection on discrete parameters. A site coordinator organizes the activity of each CoPE site. This section will describe the basic activities of a $\mathrm{CoPE}$ along with descriptions how to perform them.

\subsection{Creation of a document}

As previously mentioned, information in CoPE is represented in the form of documents. A document could contain any kind of information that the group might find useful and make decisions on. It could be a proposal, meeting minutes, or an article. In any case, creating a document in CoPE is an essential task.

Once a user is logged in and authenticated to the CoPE, he will see a screen like Figure 1. On the left side of the screen is the navigation tree. The navigation tree is a visual representation of the structure and organization of the portal. The portal's content is organized in a hierarchical folder structure similar to what is found in most operating systems such as Windows or Mac OS. The first step in creating a document is to find the folder in which the document should reside. When a folder has been selected, a window appears that allows the user to create a document or subfolder. A document is created by either entering text in the provided textbox or by uploading a Microsoft Word or PDF file. When a 
document is first created it is in a 'draft' state. This is the initial state of the document's workflow as shown in figure 2. When a document is in 'draft' state it is only visible to the creator of the document.

\subsection{Sharing a document}

When a document is first created it is in 'draft' state. In this state, the document is only visible to the creator and not visible to the group. The 'draft' state allows the creator to further edit and refine the document without it being observed by the group. When the user feels that their document is complete and ready to share, the user can make their document visible to the entire group by submitting it. Submitting a document is done by clicking on the 'submit' action button. Clicking on the 'submit' button puts the document in to a new state called the 'submitted' state. This transition can be seen in the workflow diagram provided in figure 2. In this state, the document is visible to the entire group.

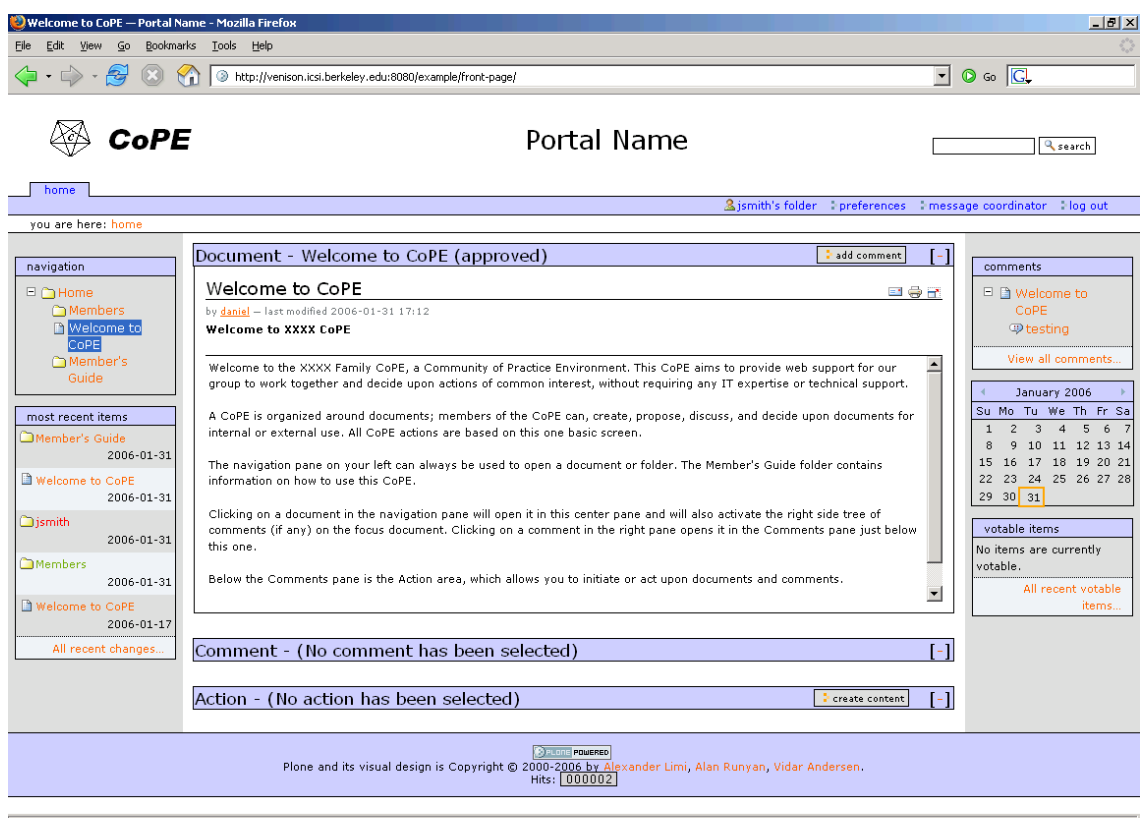

Figure 1: $\quad$ Screenshot of a user who is logged in.

\subsection{Discussion on a document}

Groups that have the ability to do so will often hold physical meetings to discuss matters and make decisions. Discussion helps the group to make better decisions by the sharing of opinions, assessing the pros and cons of an idea, and by bringing up new information. In a similar fashion, discussions in CoPE are useful for group members to exchange opinions and ideas on a document. 
Once a document is in 'submitted' state and shared with the group, users can have a discussion on the document by adding comments. This is done by clicking on the 'add comment' action button and entering a comment in a textbox. Comments for a selected document are located on the right side of the screen in the comments tree. This tree displays the title of each comment in a hierarchical tree structure. The comment tree can be seen in figure 1. A user can view the content of a comment by clicking on the title of the comment.

\subsection{Making a decision on a document}

Once a document has been discussed, there comes a time when a decision can be made on it. CoPE uses a democratic model of decision making. This means that the document is approved or disapproved based on a vote by the group members.

When a document is in 'submitted' state, the coordinator of the site can call a vote on the document. To setup voting on a document, the coordinator clicks on the 'call vote' action button. This brings up a form that asks for the vote end date and the form of voting. The vote end date is the day when voting on the document should end. There are a variety of voting modes; the simplest just sets the number of positive votes required for the document to be accepted or 'approved'. When the document is being voted on, it is in the 'voting' state of the workflow. When a document receives the required amount of votes, it transitions from the 'voting' state to the 'approved' state. There are also facilities for archiving and reconsidering documents. The complete workflow for CoPE documents is shown in figure 2 .

\section{CoPE Workflow}

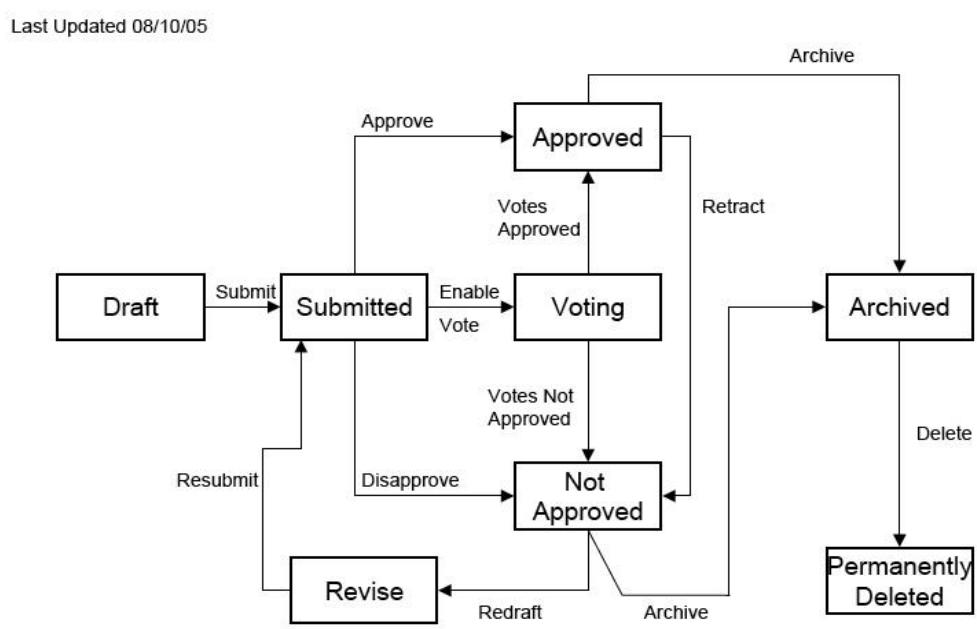

Figure 2: CoPE Workflow for documents. 'Draft' is the initial state. 


\subsection{Customization}

Different groups have different organizational models and business processes. Also, their way of doing work may change over time. This is why it is important that CoPE is able to adapt and be customized to a group's needs. To accommodate for work process differences between groups, CoPE identifies a set of characteristics that can be customized by a reasonably small set of parameters. By selecting on these parameters, an individual organization can adapt the environment to their workflow.

Customization of CoPE is done by the coordinator clicking on 'configure' in the top bar. Some of the customizations available are the mode of voting, whether votes are public, restricting the privileges of the coordinator, and the way membership approval is handled. The coordinator can select the desired setting for each.

\section{Advantages and limitations of CoPE}

\subsection{Accessibility}

Many groups are not able to use a web-based collaboration system because there are accessibility barriers. A major barrier is the lack of technical expertise to setup and maintain a site and the lack of monetary funds to acquire such expertise. This barrier is common for small non-profit groups and organizations. CoPE is designed with these types of groups in mind so it aims to make the user experience as least technical as possible. The installation and operation of CoPE should require no advanced technical skill. The ability to navigate the Internet using a web-browser should suffice.

CoPE makes the user experience smoother for the non-technical population by providing a simple interface. Many web-based collaboration system interfaces are created and designed by technologically savvy programmers. As a result, interfaces are filled with technical jargon and they are unnecessarily complex. These interfaces are geared toward providing advanced functionality and efficiency instead of focusing on usability. The interface for CoPE attempts to eliminate technical jargon and make basic tasks easy to perform. In addition, CoPE provides online documentation on how to perform various tasks so that users may reference documentation if they need assistance.

\subsection{Flexibility}

Different organizations will have varying work processes and needs. Many computer supported collaborative work systems are rigid in their structure and cannot fit the needs of all groups. These rigid collaboration systems force the group to organize and process their work in a certain way. Technology should not force groups to change their workflow and operation. Instead, technology should help and assist groups to continue their work in a more effective manner. CoPE allows more flexibility for groups by allowing them to customize the system based on a pre-determined set of parameters such as the voting mode for 
documents. Some of the parameters available for the voting mode are 'threshold' (fixed number of approval votes to approve document), 'majority' (over half of group members approve), and 'unanimity' (no negative votes with at least one half quorum of membership). Groups can choose their desired voting mode based on their workflow and style of governance.

\subsection{Governance and decision making}

CoPE provides groups with the ability to make decisions on documents. The governance and decision making style of CoPE is democratic. This concept is most obviously seen in the document voting process. The decision and power to approve a document is given to the members of the group. Each CoPE site has a coordinator who oversees the site and has special privileges. The coordinator has the ability to customize the site and can have the power to approve documents without a vote. However, the activities of the coordinator are traced and available to members. Because of this, the group can hold the coordinator accountable. If the coordinator takes any objectionable actions, the members can take appropriate measures to reverse the action or even ask for the coordinator to step down from his/her role. This democratic model of governance and decision making can help the group to establish leadership and coordination while preventing the site from being dominated by one member.

\subsection{Implementation}

CoPE is built by modifying an open source content management system called Plone [2] (http://www.plone.org). Plone's open source nature and large user/developer population made it a good fit as a base for developing CoPE. Plone provides most of the content management technology needed by CoPE, such as database storage, user authentication, and workflow. CoPE adds and modifies code in Plone to achieve desired functionality. In addition, CoPE hides and disables many features in Plone that are unnecessary. Presentation code in CoPE is written in HTML and CSS while back-end logic is written in Python [3] (http://www.python.org). CoPE also uses JavaScript for dynamic aspects of the site such as the navigation tree, comment tree, and collapsible panes. The current version of Plone that CoPE uses is version 2.1. Plone is built on top of an application server called Zope [4] (http://www.zope.org).

Currently, all CoPEs are hosted by a server from the International Computer Science Institute (http://www.icsi.berkeley.edu) running on a Linux platform. However, it is possible to run CoPE from almost all major platforms including Windows, Mac OS X, and Unix.

\subsection{Limitations on CoPE}

The CoPE system combines a collection of features not found in any system, certainly not in one that is freely available and usable by non-experts. But its capabilities overlap with many different kinds of existing systems and it is not yet clear what kinds of groups will adopt it. As with any system, there is an 
initial barrier - it does require some effort to learn to use a CoPE and this cannot be totally eliminated.

A CoPE is most useful for a group that cannot easily meet in person, but needs to produce results and decisions expressed as documents. For casual interchanges there are easier systems. Wikis are a better way to organize a space with complete user autonomy. Word processors have better tools to facilitate multiple editors working concurrently on the same document. There are much more ambitious systems to help community organizations with web design, fund raising, and similar activities. None of these currently provides the central functionality of CoPE. One possibility, however, is that it will become part of other platforms.

\section{Application experience}

The CoPE system has been deployed since the fall of 2004 and has been used by a variety of groups. As usual, the system has been undergoing modification as we learn more about the task. We briefly describe our experience with four rather different applications.

\subsection{Community organizations in California's Central Valley}

One of the ongoing CoPE applications involves the executive committee of a partnership of some twenty separate community organizations in California's Central Valley. These organizations are spread over great distances and not all of the members are comfortable in English. They have only been able to meet in person occasionally and even a phone meeting involves considerable trouble and expense.

This group was one of the initial users of our first, much more primitive, CoPE platform and has migrated to the new system described in this paper. The coordinator is the Executive Secretary of the group and has no special IT expertise. The new CoPE has not yet become the primary mechanism of discussion and decision, but it might. There is also an effort to exploit the new bi-lingual capability of CoPE.

\subsection{Faculty meetings at a research university}

Another use of the previous CoPE platform was by a group of faculty in an interdisciplinary educational program at a research university. This group was physically co-located, but it was still difficult to schedule meetings that everyone could attend. A related problem was that any decision taken at a meeting needed to be confirmed by an email vote.

For the academic year 2004-2005, this program held all of its meetings and discussions using a CoPE, except for one critical discussion of hiring. There seemed to be general agreement that it went well, but the group has not converted to the new system. This appears to be because there is very little common effort or decision making and almost no group discussion, so the 
occasional email ballot suffices. There is some overhead in remembering how to use a $\mathrm{CoPE}$ and occasional groups may well not want to bother.

\subsection{Workshop on linguistic theory}

A rather different academic application of CoPE was carried out in the summer of 2005 in conjunction with the annual Summer Institute of Linguistics. A twoday workshop explicitly tried to bring together invited speakers from a very wide range of perspectives on a topic of fundamental interest. A standard problem with such workshops is that people often spend most of the time learning about various perspectives, leaving little time for discussion.

For this workshop, all of the invited speakers were invited to submit a paper (some sent more) to be posted for discussion on a CoPE, some months in advance. In addition, the workshop and its CoPE were announced to the general linguistics community and comments were invited. There was less open discussion than we expected, but the workshop was unusually productive and apparently was the most popular of the dozens offered. This was not a continuing meeting so the CoPE was disbanded, but there seems to be great prospects for maintaining a CoPE for continuing discussions in a discipline.

\subsection{Outreach for diversity in IT}

One currently active CoPE involves a community-based effort to increase diversity among young students entering information technology and other scientific fields. The organization runs a summer program in IT for students of color and young women and also has programs to help their families understand the higher education system. These summer programs are augmented by monthly meetings during the school year.

This outreach effort is using CoPE to try to help with a number of difficulties in extending their program. For the technical content component, web-based instruction can greatly extend the number of students reached while still providing expert advice. But much of the learning happens in discussions among students and we are seeing if a dedicated CoPE can support similar discussion among physically separated students. Similarly, the family counselling aspect of this program relies heavily on interaction among families. The current experiment will test if a second CoPE for the families can help develop a community of practice in supporting underrepresented group students.

\section{Conclusions}

Despite the vast amount of online interchange, there is very little productive work done outside established institutional frameworks. The obvious exception is the open source software movement, manifestly a group of IT experts. The CoPE project began as an exploration of how groups without such IT expertise could work cooperatively over the web.

A rather complete survey in 2003 failed to reveal any freely available systems for this purpose and further analysis in 2006 confirms this finding. However, it 
has recently been discovered, at the writing of this paper (2006), that there is a project called Deme [5] led by Todd Davies at Stanford University that is similar in nature to CoPE. In any event, we have built and deployed CoPE, a web based platform for cooperative work by informal groups that might lack IT expertise and the resources to purchase it. The system has proved useful in a range of different settings and is being tried in others. The initial start up is still harder than it should be and currently requires some direct interaction. Much work remains to be done, but we believe that we have shown that an open, userfriendly, platform for cooperative work is feasible and could have a profound effects.

\section{References}

[1] Weber, S., The Success Of Open Source, Harvard University Press, 2004.

[2] Plone: A User-friendly and Powerful Open Source Content Management System, http://www.plone.org

[3] Python Programming Language, http://www.python.org

[4] Zope, http://www.zope.org

[5] Davies, T., O’Connor, B., Cochran, A.A. \& Effrat, J., An Online Environment for Democratic Deliberation: Motivations, Principles, and Design, http://www.groupspace.org, 2004.

[6] McKay, A., The Definitive Guide to Plone, Springer-Verlag: Heidelberg and New York, 2004. 